\title{
Emergence of a spectral gap in a class of random matrices associated with split graphs
}

\author{
Kevin E. Bassler ${ }^{1,2,3,4}$ and R.K.P. Zia Zi,5,6 $^{4,5}$ \\ ${ }^{1}$ Department of Physics, University of Houston, Houston, TX 77204, USA. \\ 2 Department of Mathematics, University of Houston, Houston, TX 77204, USA. \\ ${ }^{3}$ Texas Center for Superconductivity, University of Houston, Houston, TX 77204, USA. \\ ${ }^{4}$ Max-Planck-Institut für Physik komplexer Systeme, Nöthnitzer Str. 38, Dresden D-01187, \\ Germany \\ ${ }^{5}$ Center for Soft Matter and Biological Physics, Physics Department, Virginia Polytechnic \\ Institute and State University, Blacksburg, VA 24061, USA \\ ${ }^{6}$ Department of Physics and Astronomy, Iowa State University, Ames, IA 50011, USA \\ E-mail: bassler@uh.edu,rkpzia@vt.edu
}

\begin{abstract}
Motivated by the intriguing behavior displayed in a dynamic network that models a population of extreme introverts and extroverts (XIE), we consider the spectral properties of ensembles of random split graph adjacency matrices. We discover that, in general, a gap emerges in the bulk spectrum between -1 and 0 that contains a single eigenvalue. An analytic expression for the bulk distribution is derived and verified with numerical analysis. We also examine their relation to chiral ensembles, which are associated with bipartite graphs.
\end{abstract}




\section{Introduction}

The properties of ensembles of random matrices, particularly the spectra of their eigenvalues, are important for many applications and have been the basis of foundational studies in fields ranging from physics [1] to statistics [2] to ecology [3, 4]. They have also been of broad interest in both theoretical physics and pure mathematics [5, 6]. Among the most notable results in random matrix theory is the Wigner semi-circle law [7, 8]. It applies to classes of matrices including real symmetric matrices and complex hermitian matrices with i.i.d. random diagonal elements and i.i.d. random off-diagonal elements, which can be different distributions, so long as the moments of the off-diagonal distribution, up through the fourth one, are finite [9]. For such matrix ensembles, Wigner's law says that in the large matrix limit, the eigenvalue probability distribution function (epdf) is a semi-circle. Similar results apply to other classes of random matrices. The singular values of asymptotically large rectangular matrices having i.i.d. random elements with finite variance are distributed according to the Marchenko-Pastur law [10]. Also, the eigenvalues of chiral random matrices come in plus/minus pairs, but have a semi-circle pdf in the large matrix limit [6]. Low-rank perturbations of random matrices, however, can cause epdfs to deviate from these semi-circle type laws in interesting ways when the matrices are finite [6, 11, 12].

In network science, eigenvalues of ensembles of random matrices are used to characterize the structure and properties of classes of networks, or graphs [13, 14, 15]. Spectra can reveal structural properties including modularity [16, 17] and existence of motifs [18, 19, 20] that affect dynamical properties including synchronizability [21] and redundancy [22]. Adjacency and Laplacian matrices both provide complete descriptions of the structure of networks. Studies of ensembles of random ensembles adjacency matrices have shown that the largest eigenvalue typically separates from the bulk distribution [23] due to the non-zero mean value of the elements [24, 25, 26] and that the bulk of the eigenvalue distribution of Erdős-Rényi networks [27] follows the semi-circle law in the limit of infinite mean degree [28, 29], but that it can have a different, "triangle-like" distribution with power-law tails when the degree of the nodes are power-law distributed [23, 30].

In this context, our recent interest in a class of non-equilibrium dynamical models of social networks motivated us to study the ensembles of their associated adjacency matrices. In these models [31, 32, 33, 34, 35], a node (an individual in a social setting) may add or cut edges (contacts with other individuals) according to some "preference." Such propensities may be internal, such as introverts preferring few contacts and extroverts, many. There may also be external circumstances which affect how many contacts an individual might "prefer," e.g., during a raging epidemic. Assuming time independent probabilities for individuals to cut/add links, the system will come into steady state, leading to a time-independent ensemble of adjacency matrices, $\mathbb{A}$. Typically, the dynamics governing the evolution of such systems do not obey detailed balance [34] and so, the stationary distribution, $\mathcal{P}^{*}(\mathbb{A})$ is not known, so that Monte Carlo simulations provide the only way to proceed. Remarkably, there is a special limit where detailed balance holds and an explicit $\mathcal{P}^{*}(\mathbb{A})$ is found [33]. Known as the XIE model, this limit consists of a population of extreme introverts (I) and extroverts (E), in which an I, 
when chosen, cuts a randomly chosen link while a chosen $\mathrm{E}$ adds a link to a random individual not already connected to it. As a result, the A's reduce to blocks, corresponding to no I-I links, fully connected E-E group, and a dynamic set of I-E cross-links.

In the context of graph theory, such networks are associated with split graphs [36, 37], with many interesting properties. Thus, our focus here can also be phrased as "properties of the spectra of split graph adjacency matrices." To be specific, consider the ensemble of $2 \mathrm{~N} \times 2 \mathrm{~N}$ matrices $\mathbb{A}$ of the form

$$
\mathbb{A}=\left(\begin{array}{cc}
\mathbb{D} & \mathbb{X} \\
\mathbb{X}^{T} & \mathbb{M}
\end{array}\right)
$$

where $\mathbb{X}$ is an $N \times N$ square matrix with i.i.d. random Boolean variables ( 0 or 1$)$ as elements and $\mathbb{Q}$ is a matrix with only 0 elements. Here,

$$
\mathbb{M}=-\llbracket+|u\rangle\langle u|
$$

where $|u\rangle$ is the $(N \times 1)$ vector with unity in every element $f\langle u|=| u\rangle^{T}$. Matrices of this form appear naturally in "critical" XIE networks [38, 39], i.e., ones with with equal numbers $(N)$ of introverts and extroverts. Thus, we will refer to matrices of the above form $X I E$ matrices. To reiterate, the 0 block diagonal matrix corresponds to the adjacency matrix of the I's, $\mathbb{M}$ the adjacency matrix of the E's, and $\mathbb{X}$ the incidence matrix of the I-E pairs. Now, there are non-trivial correlations among the I-E cross-links, so that the elements of $\mathbb{X}$ are not i.i.d. The differences between the epdf of such adjacency matrices and that of random split graphs should reflect the correlations between the elements in $\mathbb{X}$. In this paper, as a first step, we restrict consideration to random split graphs.

Closely related to split graphs are bipartite graphs, corresponding to a network with only I-E links. The associated adjacency matrices are known as chiral matrices\$. The principal finding reported here is the emergence of a gap in the epdf of such matrices when "M is added," i.e., when a bipartite graph is connected to form a split graph (or when one of the two sets of independent nodes are linked to form a clique). In particular, for chiral matrices with random Boolean variables ( 0 or 1 ) of equal probability as elements, the epdf is symmetric around 0 , with the bulk obeying the simple semi-circle law, apart from isolated ev's far outside [26, 43]. By contrast, Fig. 1 1 shows the striking epdf associated with the latter, reminiscent of a (upside down) Viking ship or the steeple of the Stykkishólmskirkja. Many different features are evident: asymmetry, serious distortions from semi-circle, peaks at -1 and 0 , and a gap between them. It appears as if the negative part of the distorted semi-circle were shifted to more negative values, while the isolated eigenvalues on edge of the bulk appears to be shifted to more positive values! Additionally, careful examination shows a single eigenvalue lying within the gap (close to -1 here). The two eigenvalues separated from the bulk appear as isolated peaks (near -22

$\ddagger$ Note that, for any matrix $\mathbb{A},\langle u|\mathbb{A}| u\rangle$ is the sum over all its elements.

$\S$ In most of the literature, chiral matrices are of the form $\left(\begin{array}{cc}\mathbb{D} & \mathbb{X} \\ -\mathbb{X}^{T} & \mathbb{O}\end{array}\right)$. The ones here differ only by a sign, i.e., $\left(\begin{array}{cc}\mathbb{0} & \mathbb{X} \\ \mathbb{X}^{T} & \mathbb{0}\end{array}\right)$. The two are clearly intimately related, with properties that can be mapped from one to the other. We follow the terminology of [6] where the latter are referred to as chiral matrices. 


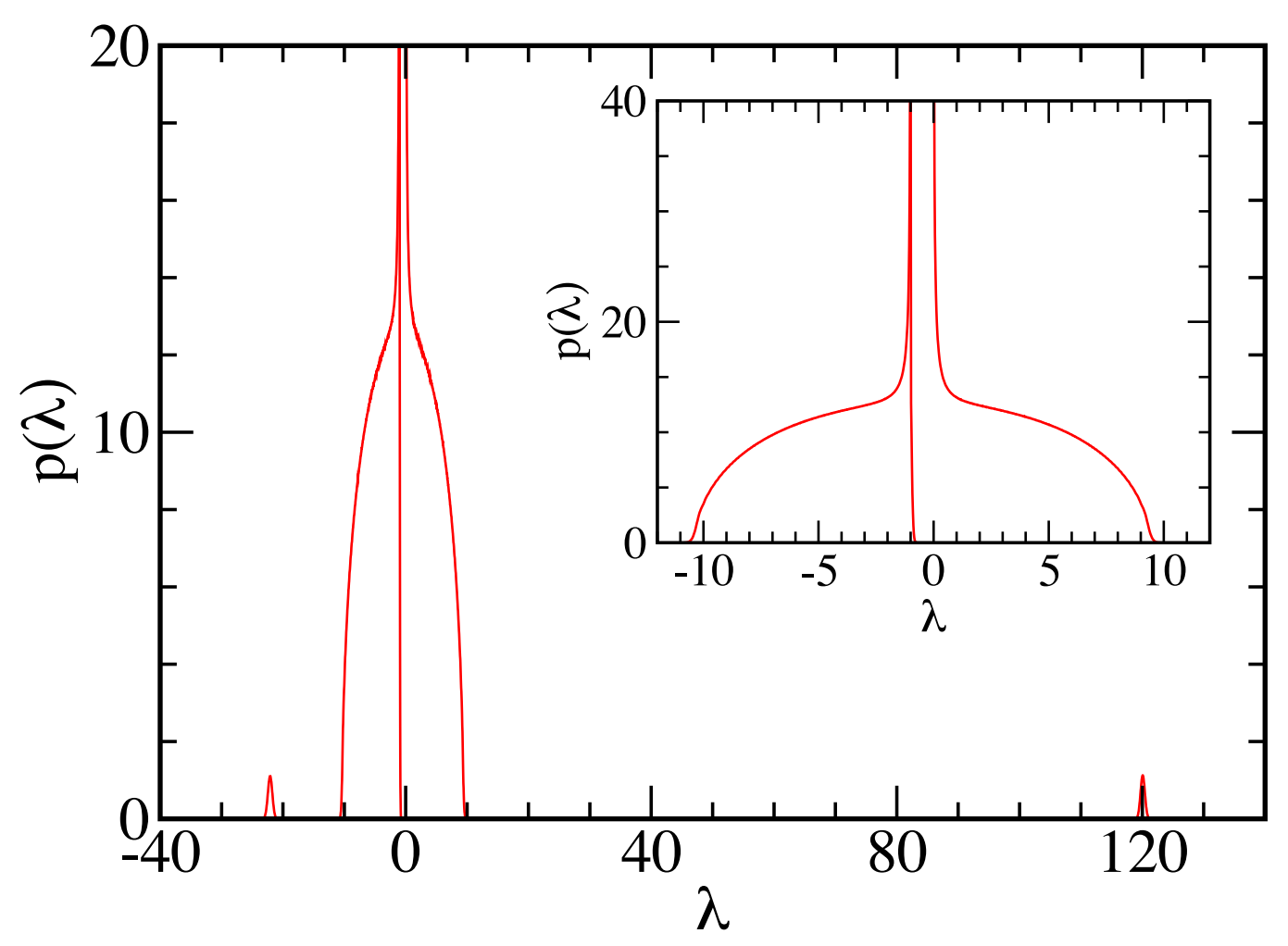

Figure 1. Eigenvalue probability distribution function of $2 N \times 2 N$ adjacency matrices of random split graphs with $N=100$ and I-E connection probability of 0.5 . Note the two separated single eigenvalues, near -22 and 120. Inset shows an enlarged view of the bulk distribution, which resembles the steeple of Stykkishólmskirkja. A gap between -1 and 0 is clearly visible. Further, there is a single eigenvalue "tightly bound" to -1 (in this case), a fact evidenced by the almost vertical line at the left edge of the gap.

and 120 here). By contrast, the eigenvalue in the gap appears "tightly bound" to the left edge, so that the pdf descends rapidly as $\lambda$ increases from -1 . For more generic $\mathbb{X}$ 's (e.g., different means), this eigenvalue can be detached from both ends and isolated. All of these remarkable features will be examined and explained. Random split graphs are a type of stochastic block model [40, 41, 42] for which methods of spectral analysis are well developed [17]. However, those methods require a finite variance for the elements of the blocks. The elements of the I-I and E-E blocks of split graph adjacency matrices have zero variance and, so, require other methods of analysis.

The remainder of this paper is organized as follows. We begin by establishing the connection between the adjacency matrices of bipartite and a split graphs, and their relation to chiral matrices. Through these connections, many of the features shown in Fig. 1 can be understood and, in the case where $\mathbb{X}$ consists of i.i.d. Gaussian variables of zero mean, the epdf can be computed analytically (in the large $N$ limit). We then present simulation results 


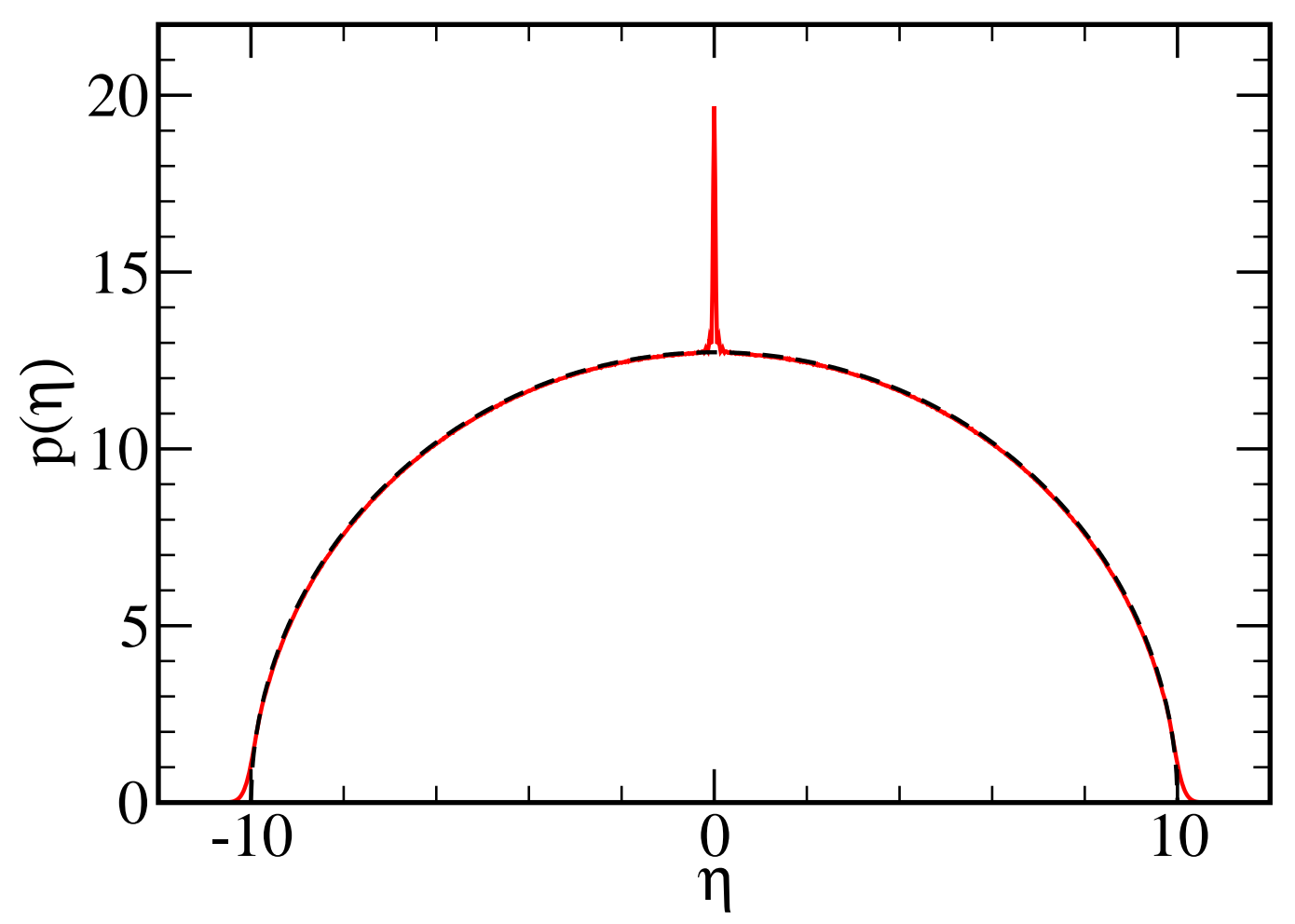

Figure 2. Eigenvalue probability distribution function of $2 N \times 2 N$ random chiral matrices with $N=100$ and Gaussian distributed elements with zero mean and variance of 0.25 . Black dashed line is the corresponding Wigner semi-circle prediction.

for various generalizations: shifted mean Gaussians, Boolean $(0,1)$ distributions, and split graph adjacency matrices. We end with a brief summary and outlook for further studies. The Appendix is devoted to some details associated with special cases.

\section{Results}

This section is devoted to establishing the connection between the spectra of chiral and XIE matrices, i.e., matrices of the form (4 blocks of $N \times N$ matrices)

$$
\mathbb{A}_{B}=\left(\begin{array}{cc}
\mathbb{D} & \mathbb{X} \\
\mathbb{X}^{T} & \mathbb{Q}
\end{array}\right) \quad \text { and } \quad \mathbb{A}_{S}=\left(\begin{array}{cc}
\mathbb{D} & \mathbb{X} \\
\mathbb{X}^{T} & \mathbb{M}
\end{array}\right) .
$$

When $\mathbb{X}$ has Boolean elements, these forms correspond to adjacency matrices of, respectively, bipartite graphs (B) and split graphs (S). However, in what follows, we also consider matrices with $\mathbb{X}_{s}$ that have Gaussian distributed elements. We begin with finding the connection between these matrices for a specific $\mathbb{X}$. Then, we consider ensembles of them with random i.i.d. elements, first examining Gaussians distributions with zero mean and then exploring 
ones with positive mean, specifically, with Boolean elements.

\subsection{Considerations for a specific $\mathbb{X}$}

First, consider a particular $\mathbb{X}$ (as opposed to an ensemble of them), and denote the eigenvectors of $\mathbb{A}_{B}$ by the doublet of $N \times 1$ vectors along with eigenvalues $\eta_{\alpha}$

$$
\mathbb{A}_{B}\left(\begin{array}{l}
\left|\tilde{w}_{\alpha}\right\rangle \\
\left|w_{\alpha}\right\rangle
\end{array}\right)=\eta_{\alpha}\left(\begin{array}{l}
\left|\tilde{w}_{\alpha}\right\rangle \\
\left|w_{\alpha}\right\rangle
\end{array}\right)
$$

Thus,

$$
\mathbb{X}\left|w_{\alpha}\right\rangle=\eta_{\alpha}\left|\tilde{w}_{\alpha}\right\rangle ; \mathbb{X}^{T}\left|\tilde{w}_{\alpha}\right\rangle=\eta_{\alpha}\left|w_{\alpha}\right\rangle
$$

so that

$$
\mathbb{W}\left|w_{\alpha}\right\rangle \equiv \mathbb{X}^{T} \mathbb{X}\left|w_{\alpha}\right\rangle=\mu_{\alpha}\left|w_{\alpha}\right\rangle
$$

with

$$
\mu_{\alpha} \equiv \eta_{\alpha}^{2} ; \quad \alpha=1, \ldots, N
$$

Note that $\mathbb{W}$ is referred to as a Wishart matrix [6] [I]. For later convenience, we will assume the $w$ 's are normalized, so that any function of the matrix $\mathbb{W}$ can be written as

$$
f(\mathbb{W})=\sum_{\alpha} f\left(\mu_{\alpha}\right)\left|w_{\alpha}\right\rangle\left\langle w_{\alpha}\right|
$$

We will also focus on the generic case where there are no degeneracies and strictly positive $\mu$ 's: $0<\mu_{1} \leq \mu_{2} \leq \ldots \leq \mu_{N}$. Exceptions to these will be deferred to the Appendix. From here, it is easy to see that the $2 N \eta$ 's come in $N$ plus/minus pairs, which we label by $\eta_{\alpha \pm}= \pm \sqrt{\mu_{\alpha}}$. Associated with these are eigenvectors (of $\mathbb{A}_{B}$ ) in the form of a doublet: $\left(\mathbb{X}\left|w_{\alpha}\right\rangle / \eta_{\alpha \pm},\left|w_{\alpha}\right\rangle\right)$.

Proceeding, we consider the eigenvectors and eigenvalues of $\mathbb{A}_{S}$ :

$$
\mathbb{A}_{S}\left(\begin{array}{l}
|\tilde{v}\rangle \\
|v\rangle
\end{array}\right)=\lambda\left(\begin{array}{l}
|\tilde{v}\rangle \\
|v\rangle
\end{array}\right)
$$

Consider the case $\lambda \neq 0$ first so that we may use $|\tilde{v}\rangle=\mathbb{X}|v\rangle / \lambda$ to eliminate $|\tilde{v}\rangle$ as before and arrive at

$$
\mathbb{W}|v\rangle / \lambda+\mathbb{M}|v\rangle=\lambda|v\rangle
$$

Inserting the explicit form (2), we find

$$
\mathbb{W}|v\rangle=\left(\lambda^{2}+\lambda\right)|v\rangle-\lambda c|u\rangle
$$

where $c \equiv\langle u \mid v\rangle$ is just the sum of the elements of $|v\rangle$. The solution to 10 is clear

$$
|v\rangle=\lambda c\left[\lambda^{2}+\lambda-\mathbb{W}\right]^{-1}|u\rangle
$$

Projecting onto $|u\rangle$, we have $c=\lambda c\left\langle u\left|\left[\lambda^{2}+\lambda-\mathbb{W}\right]^{-1}\right| u\right\rangle$. Assuming $\mathbb{I} \neq 0$ and exploiting (7), an explicit form for the secular equation emerges:

$$
1=\lambda \sum_{\alpha=1}^{N} \frac{c_{\alpha}^{2}}{\lambda^{2}+\lambda-\mu_{\alpha}}
$$

\| The collection of W's with random $\mathbb{X}$ 's is known as the Laguerre ensemble.

II The special cases where $\lambda$ or $c$ vanish are also studied in the Appendix. 
where

$$
c_{\alpha} \equiv\left\langle u \mid w_{\alpha}\right\rangle
$$

The right hand side can be written as a sum,

$$
1=\sum_{\alpha} \frac{c_{\alpha}^{2}}{\rho_{\alpha+}-\rho_{\alpha-}}\left[\frac{\rho_{\alpha+}}{\lambda-\rho_{\alpha+}}+\frac{\left(-\rho_{\alpha-}\right)}{\lambda-\rho_{\alpha-}}\right]
$$

over $2 N$ simple poles, at

$$
\rho_{\alpha \pm}=\frac{1}{2}\left\{-1 \pm \sqrt{1+4 \mu_{\alpha}}\right\}
$$

Since $\mu>0$, these poles are located outside the interval $[-1,0]$, while all residues are positive. Thus, as $\lambda$ is varied from $-\infty$ to $+\infty$, the right hand side begins at 0 , falls to $-\infty$ at $\rho_{N-}=\left(-1-\sqrt{1+4 \mu_{N}}\right) / 2$, runs from $+\infty$ to $-\infty$ between each successive $\rho_{\alpha \pm}$ until past

$\rho_{N+}=\left(-1+\sqrt{1+4 \mu_{N}}\right) / 2$, and finally falls from $+\infty$ to 0 . The consequence is that there is precisely one solution to (13) between successive pairs of $\rho$, plus one more beyond $\rho_{N+}$. As $\alpha \in[1, N]$, there are precisely $2 N$ solutions, which we label by $\lambda_{\alpha \pm}$. Such interlacing between $\lambda$ 's and $\rho$ 's ${ }^{+}$

$$
\rho_{N-}<\lambda_{N-}<\ldots<\rho_{1-}<\lambda_{1-}<\rho_{1+}<\lambda_{1+}<\rho_{2+1}<\ldots<\rho_{N+}<\lambda_{N+}
$$

is a familiar occurrence in random matrix theory (e.g., in [26]). Of course, it is not easy to find the precise location of each $\lambda_{\alpha}$, as details such as $\left\{c_{\alpha}\right\}$ will determine whether a $\lambda_{\alpha}$ is closer to $\rho_{\alpha}$ or $\rho_{\alpha+1}$. As will be shown below, we can nevertheless draw meaningful conclusions for many aspects of he epdfs of random matrices. Finally, the eigenvectors associated with $\lambda_{\alpha \pm}$ are the doublets: $\left(\mathbb{X}\left|v_{\alpha \pm}\right\rangle / \lambda_{\alpha \pm},\left|v_{\alpha \pm}\right\rangle\right)$, where $\left|v_{\alpha \pm}\right\rangle$ is given by $(11)$ with $\lambda=\lambda_{\alpha_{ \pm}}$.

\subsection{Ensembles of $\mathbb{X}$ with zero mean (Gaussian distributions)}

Turning from a specific $\mathbb{X}$ to ensembles of them, these considerations allow us to predict the prominent differences between the epdfs in Fig. 2 and Fig. 3, as well as to understand a number of features in Fig. 1. In particular, let us first consider the simplest case: a chiral ensemble in which $\mathbb{X}$ consists of Gaussian distributed elements with zero mean and finite variance $\sigma^{2}$. For $N \rightarrow \infty$, the epdf obeys the celebrated semicircle law [6]: $p(\eta) \propto \sqrt{R^{2}-\eta^{2}}$ with $R=2 \sigma \sqrt{N}$. In Fig. 2, the red line shows data for the epdf of the $N=100$ case, with $\sigma=1 / 2$ and support $[-10,10]$. Apart from finite $N$ effects (e.g., tails beyond \pm 10 , visible peak at 0), they fit well into the semicircle law (black dashed line). Note that the numerical results for the epdfs in all figures were calculated from ensembles of $10^{7}$ random matrices with bin widths of $10^{-3}$. Also note that, in all figures, lines connect the discrete data points. However, we have not drawn lines to connect the data points at 0 and -1 with the points inside the gap to emphasize the discontinuity in the description of the epdf at the edges of the gap.

+ Some $\lambda$ 's may be equal to $\rho$ 's. See Appendix 1 for details. 


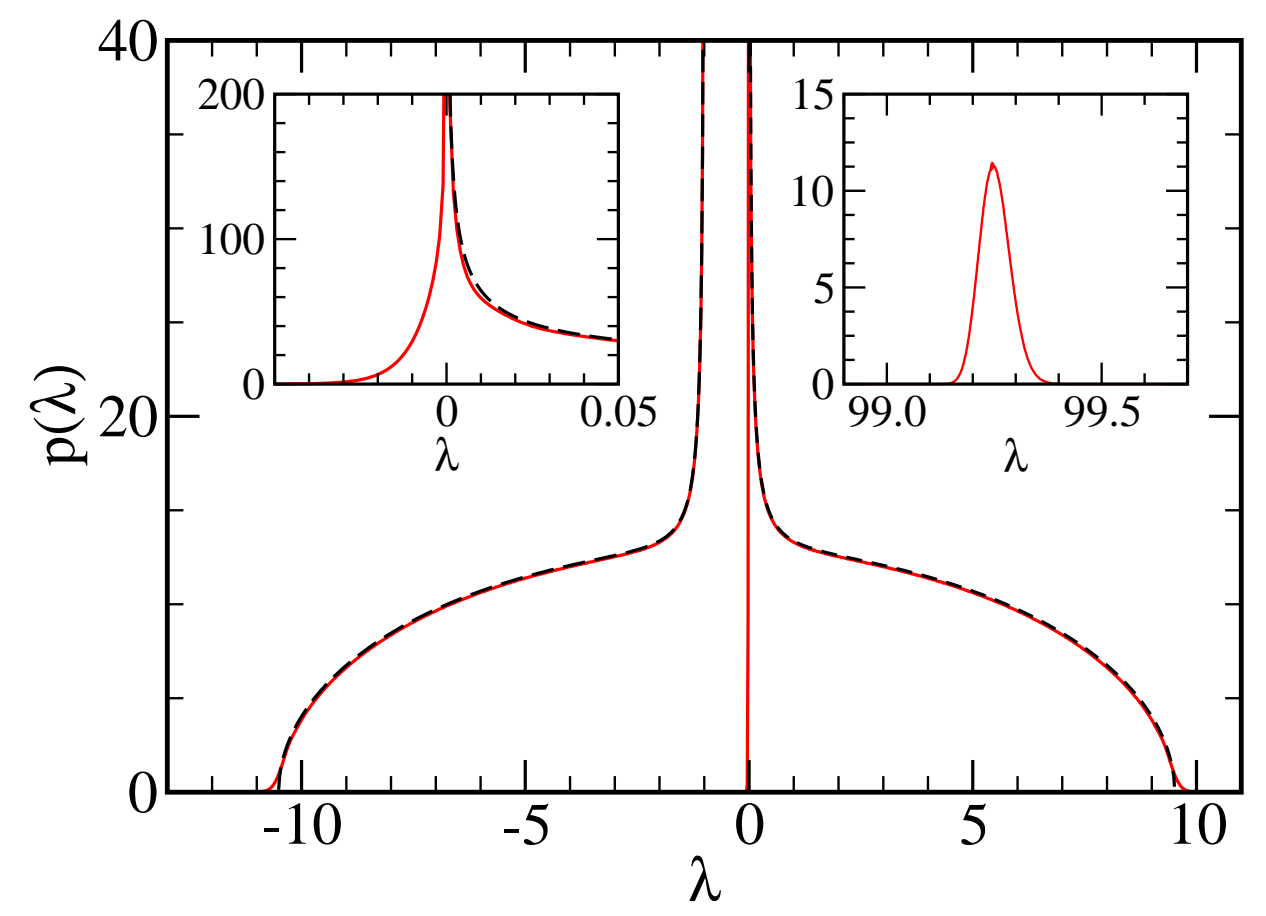

Figure 3. Eigenvalue probability distribution function of $2 N \times 2 N$ XIE matrices with $N=100$ and I-E connection probability of 0.5 and Gaussian distributed I-E elements with zero mean and variance of 0.25 . Black dashed line is our analytic prediction for the bulk distribution. Left inset shows an enlarged view of the gap region near $\lambda=0$. Note that the gap contains a single eigenvalue, which happens to be "tightly bound" to 0 in this case. The presence of this eigenvalue and its location is emphasized by the almost vertical line near the right edge of the gap in the main plot. Right inset shows the separated eigenvalue near $\lambda=99.25$

Next, we follow the analysis in Section 2.1 and consider the effects of "adding $\mathbb{M}$ " to this ensemble. In particular, for Fig. 3 shows the epdf of XIE matrices $\left\{\mathbb{A}_{S}\right\}$, using the same zero mean Gaussian ensemble of $\mathbb{X}$ as in Fig. 2. Thus, we know how the $\rho$ 's are distributed:

$$
p(\rho)=p(\eta(\rho))\left|\frac{d \eta}{d \rho}\right| \propto|1+2 \rho| \sqrt{\frac{N}{\rho^{2}+\rho}-1}
$$

This is the black dashed line plotted in Fig. 3. The emergence of the gap is now clear: The solutions to $\rho^{2}+\rho=\mu=\eta^{2} \in[0, N]$ are less than $\rho_{1-}=-1$ and greater than $\rho_{1+}=0$. The divergence at the edges of the gap (the Viking-ship like feature) is due to $\left(\rho^{2}+\rho\right)^{-1 / 2}$ in the Jacobian. Meanwhile, since the $\lambda$ 's are squeezed between successive $\rho$ 's, the distribution of the bulk values are identical as $N \rightarrow \infty$, apart from two exceptions to be discussed below. As we see in Fig. 3, there is excellent agreement between the data and this prediction: $p_{b u l k}(\lambda)=p(\rho)$. Notice that, while $p(\eta)$ vanishes at \pm 10 , the bulk of $p(\rho)$ vanishes at -10.51 and 9.51 , the solutions to $\rho^{2}+\rho=N=100$. These values provide excellent approximations to the data.

There are two exceptions to $\lambda \simeq \rho$, shown in the insets of Fig. 3 . One is the largest 
eigenvalue, $\lambda_{N+}$, which is not only greater than $\rho_{N+}=O\left(N^{1 / 2}\right)$, but much greater. If we assume $\lambda_{N}=O(N)$, then we can estimate it as follows. In $(13)$, let $1 /\left(\lambda_{N}-\rho_{\alpha+}\right) \simeq 1 / \lambda_{N}$, so that

$$
\lambda_{N} \simeq \sum_{\alpha} c_{\alpha}^{2}
$$

But, $c_{\alpha}^{2} \equiv\left\langle u \mid w_{\alpha}\right\rangle^{2}=\left\langle u \mid w_{\alpha}\right\rangle\left\langle w_{\alpha} \mid u\right\rangle$ and $\sum_{\alpha}\left|w_{\alpha}\right\rangle\left\langle w_{\alpha}\right|=\llbracket$, so that $\sum_{\alpha} c_{\alpha}^{2}=\langle u|\llbracket| u\rangle=N$. Thus, to leading order, we find

$$
\lambda_{N} \simeq N
$$

which justifies our assumption of $\lambda_{N}=O(N)$. To account for the next order, we may expand the denominators on the right in (12):

$$
\begin{aligned}
\lambda_{N}+1 & =\sum c_{\alpha}^{2}\left[1+\frac{\mu_{\alpha}}{\lambda_{N}^{2}+\lambda_{N}}+\ldots\right] \\
& =N+\frac{1}{\lambda_{N}^{2}+\lambda_{N}} \sum \mu_{\alpha} c_{\alpha}^{2}+\ldots
\end{aligned}
$$

But $\Sigma_{\alpha}\left|w_{\alpha}\right\rangle \mu_{\alpha}\left\langle w_{\alpha}\right|=\mathbb{W}$, so that the sum in the last equation is $\left\langle u\left|\mathbb{X}^{T} \mathbb{X}\right| u\right\rangle$. Averaged over the ensemble, this quantity is well approximated by the sum of the variance of each element in $\mathbb{X}$. The end result is

$$
\lambda_{N}=N-1+\sigma^{2}+\ldots
$$

which is 99.25 in the case of our simulation study. This approximation compares well with the distribution of the largest eigenvalue, shown in the right inset of Fig. 3 .

The other exception is $\lambda_{-1}$ which lies within the gap: $\left[\rho_{1-}, \rho_{1+}\right]=[-1,0]$. While a solution to 13 must exist between these $\rho$ values, finding an analytic expression of its precise location remains a challenge. As the inset on the left in Fig. 3 shows, it appears to be "tightly bound" to the right edge with probability decreasing exponentially as $\lambda$ decreases from 0 . Such behavior is opposite to the case in Fig. 1 ("tight binding" to the left edge). Below, we will return to further investigations of this eigenvalue in the gap.

\subsection{Ensembles of $\mathbb{X}$ with positive mean (Boolean distributions)}

A well-known phenomenon in random matrix theory is that, if it consists of i.i.d. random elements with non-zero mean, an isolated eigenvalue may emerge from the bulk (e.g, the semi-circle) epdf. This is certainly the case if the elements were Boolean variables, i.e., randomly 1 with probability, $q$, and 0 with probability $1-q$. The mean is $q$ and the variance is $\sigma^{2}=q(1-q)$. By choosing $q=1 / 2$ in simulations, universality guarantees that the bulk part of the epdf should, as $N \rightarrow \infty$, approach that in the Gaussian example above. The only difference is the presence of isolated eigenvalues, separated from the bulk [26], located at $\pm \eta_{N}$ ( $\simeq 50.5$ here). For the convenience of the readers, we reproduce this behavior in Fig. 4 , so that it can be compared to Fig. 1. Furthermore, such a large $\eta_{N}$ is connected with the (lower doublet of) its associated eigenvector, $\left|w_{N}\right\rangle$, being mainly along $|u\rangle$. 


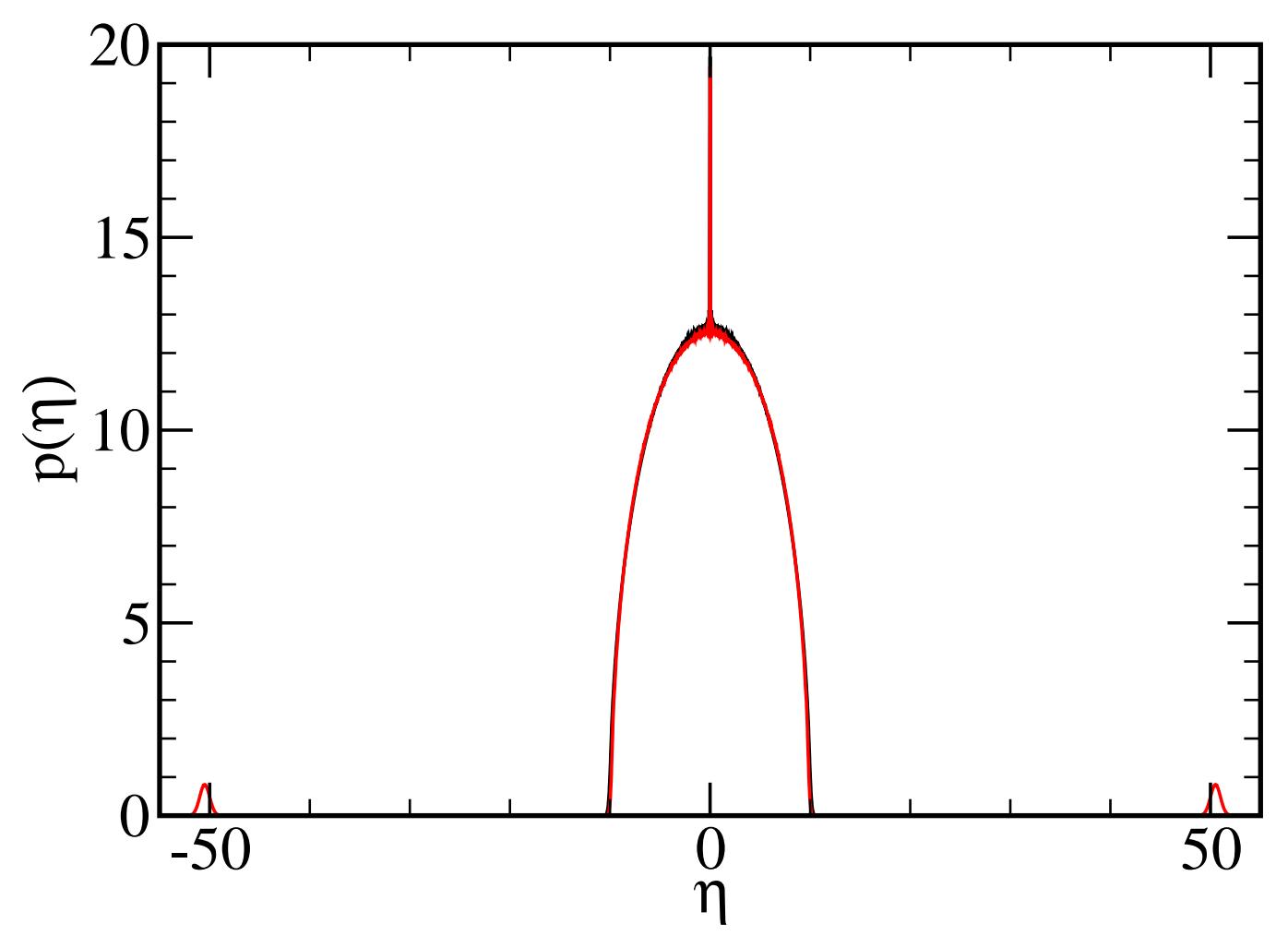

Figure 4. Eigenvalue probability distribution function of $2 N \times 2 N$ chiral matrices with $N=100$ and Gaussian distributed elements with variance of 0.25 and mean of zero (black) and of 0.5 (red). Note the two eigenvalues that separate from the bulk when the mean is non-zero.

To appreciate these connections, consider the simplest case, namely, adding mean $q \rightarrow \infty$ to $\mathbb{X}_{G}$ (the notation we use here for a zero mean Gaussian ensemble such as in Section 2.2, with variance $\sigma^{2}=O(1)$ ). Denoting $\mathbb{X}$ by $\mathbb{X}_{G}+q|u\rangle\langle u|$, we examine (6).

$$
\mathbb{X}^{T} \mathbb{X}\left|w_{N}\right\rangle=\left(\mathbb{X}_{G}^{T}+q|u\rangle\langle u|\right)\left(\mathbb{X}_{G}+q|u\rangle\langle u|\right)\left|w_{N}\right\rangle=\mu_{N}\left|w_{N}\right\rangle
$$

Clearly, to leading order, $\left|w_{N}\right\rangle \propto|u\rangle$ and $\mu_{N}=(q N)^{2}$. Normalization leads to $\left|w_{N}\right\rangle=$ $|u\rangle / \sqrt{N}$, along with $c_{N}=\sqrt{N}$. We can find the next order correction of the eigenvalue by standard means, regarding $\mathbb{V} \equiv q|u\rangle\left\langle u\left|\mathbb{X}_{G}+q \mathbb{X}_{G}^{T}\right| u\right\rangle\langle u|+\mathbb{X}_{G}^{T} \mathbb{X}_{G}$ as a perturbation for $\mu_{N}$. To first order, we find $\mu_{N}=(q N)^{2}+\left\langle w_{N}|\mathbb{V}| w_{N}\right\rangle+\ldots=(q N)^{2}+\sigma^{2} N+\ldots$. Notice that the $O(q)$ terms average to zero and so, the next non-vanishing contribution is $O(1)$. Thus, if that is to be kept, we should consider second order perturbation as well. At that order $\mathbb{V}^{2}$ contains terms $\propto(q N)^{2}$ which do not average to zero. A straightforward but tedious computation leads to another $\sigma^{2} N+\ldots$ The final result is $\mu_{N}=(q N)^{2}+2 \sigma^{2} N+\ldots$, so that $\eta_{N+}=\sqrt{\mu_{N}}=q N+\sigma^{2} / q+\ldots$. Remarkably, it is in excellent agreement with preliminary simulations studies using $N=100, \sigma=1 / 2,1,2$, and $q=1 / 2,1,2$. Of course, the typical limit of interest is large $N$ with fixed $q$, which does not necessarily commute with the large $q$ 
fixed $N$ limit here. Nevertheless, this result is the same as implicit predictions in the literature (See, e.g., [26]).

Meanwhile, since $\Sigma_{\alpha} c_{\alpha}^{2}=N$, we conclude that the sum over the rest of the $\alpha$ 's must be

$$
\Sigma^{\prime} \equiv \sum_{\alpha=1}^{N-1} c_{\alpha}^{2}=O(1)
$$

Thus, on the average, each $c_{\alpha}^{2}$ is expected to be $O(1 / N)$. As $c_{\alpha}=O\left(N^{-1 / 2}\right)$ is the projection of $\left|w_{\alpha}\right\rangle$ onto $|u\rangle$, we conclude the obvious, i.e., the rest of the eigenvectors lie mainly in a subspace orthogonal to $|u\rangle$.

Finally, we turn to the understanding of the epdf in Fig. 1. One possible route is to consider the effects of adding a mean to the $\mathbb{X}_{G}$ 's in (the off diagonal blocks of) into the $\mathbb{A}_{S}$ associated with Fig. 3. Here, we purse the easier route, examine the effects of "adding $\mathbb{M}$ " to the $\mathbb{A}_{B}$ 's associated with Fig. 4 (i.e., from adjacency matrices of bipartite graphs with Boolean $\mathbb{X}$ 's to the same for split graphs). We will find that most of striking features in Fig. 1 can be understood along the these lines as in Sec. 2.2 above.

First, as expected, the dominant part of the epdf - the bulk - are affected in much the same way that Fig. 2 is transformed into Fig. 3. Thus, we see that this part of $p(\lambda)$ vanishes outside $\simeq-10.51$ and $\simeq 9.51$, as well the presence of a gap between $\simeq-1$ and 0 . Turning to the separated eigenvalues outside, note that their distributions peak near -22.10 and 120.06. Both are quite far from the \pm 50.5 values in Fig. 4. To provide a good estimate for these, we turn again to (13), but must account for the separated eigenvalues in Fig. $3\left(\eta_{N_{ \pm}}\right)$being $O(N)$, instead of $\eta_{N_{ \pm}}=O\left(N^{1 / 2}\right)$ in Fig. 2. Starting with Eqn 12 and seeking a $\lambda_{N}=O(N) \gg \eta_{\alpha<N}$, we find

$$
\begin{aligned}
1 & =\lambda_{N} \sum \frac{c_{\alpha}^{2}}{\lambda_{N}^{2}+\lambda_{N}-\mu_{N}}=\lambda\left\{\frac{c_{N}^{2}}{\lambda_{N}^{2}+\lambda_{N}-\mu_{N}}+\sum_{\alpha=1}^{N-1} \frac{c_{\alpha}^{2}}{\lambda_{N}^{2}+\lambda_{N}-\mu_{\alpha}}\right\} \\
& =\frac{\lambda_{N}\left(N-\Sigma^{\prime}\right)}{\lambda_{N}^{2}+\lambda_{N}-\mu_{N}}+\frac{\Sigma^{\prime}}{1+\lambda_{N}}=\frac{\lambda_{N} N}{\lambda_{N}^{2}-\mu_{N}}+O\left(\frac{1}{N}\right)
\end{aligned}
$$

Substituting the leading order approximation for $\mu_{N}$, and solving $\lambda_{N}^{2}-\lambda_{N} N-q^{2} N^{2}=0$, we arrive at

$$
\lambda_{N \pm}=\frac{N}{2}\left[1 \pm \sqrt{1+4 q^{2}}\right]
$$

which are $N(1 \pm \sqrt{2}) / 2 \simeq-20.7,120.7$ for the Boolean case. These are remarkably close to the observed values, given that we took into account only the leading order!

Second, there should be an eigenvalue between $\eta_{(N-1)+}$ and $\eta_{N+}$, but the data implies it is "tightly bound" to the bulk. This aspect can be understood by the following illustration. Consider a secular equation with just two terms, $1=r_{1} /\left(\lambda-\rho_{1}\right)+r_{2} /\left(\lambda-\rho_{2}\right)$, and let $r_{1}=O(1 / \sqrt{N}), \rho_{1}=O(\sqrt{N})$, while $r_{2}, \rho_{2}=O(N)$. A simple plot of the right will provide an intuitive picture for the behavior of solutions. A recursive form

$$
\begin{aligned}
\lambda_{i} & =\rho_{i}+r_{i}+r_{j \neq i}\left(\lambda-\rho_{i}\right) /\left(\lambda-\rho_{j \neq i}\right) \\
& \simeq \rho_{i}+r_{i}+r_{j \neq i}\left(r_{i}+\ldots\right) /\left(\rho_{i}+r_{i}-\rho_{j \neq i}+\ldots\right)
\end{aligned}
$$




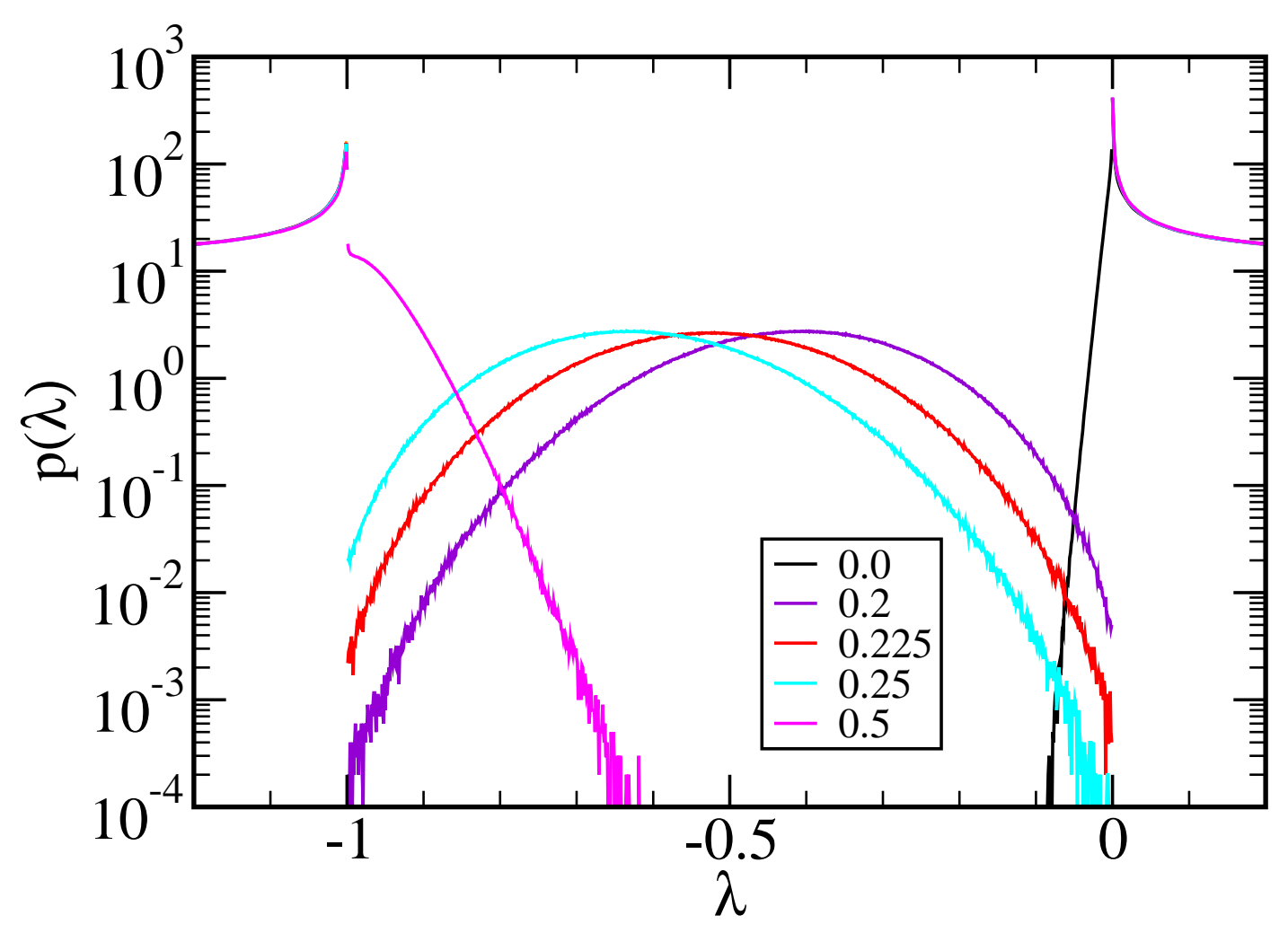

Figure 5. The effect of the mean value (of the i.i.d. random matrox elements) on the probability distribution function of the gap-eigenvalue, $\lambda_{1-} \in[-1,0]$. (Legend lists the mean values.) These studies are based on 200x200 XIE matrices with Gaussian distributed elements of variance of 0.25 .

provides a more quantitative estimate, as we see the effect of our assumptions, namely, $\lambda_{1}=\rho_{1}+O(1 / \sqrt{N})$ being "tightly bound" to $\rho_{1}$, while $\left.\lambda_{2}=\rho_{2}+r_{2}+\ldots\right)$ can be far from $\rho_{2}$.

What remains is perhaps the most challenging task: predicting the location and distribution of the eigenvalue in the gap, $\lambda_{1-}$. As noted in the illustration above, the location of the solutions depend on the details of the parameters. In our case, there are many poles nearby and a range of residues, so that $\lambda_{1-}$ can be anywhere between $\rho_{1-} \rightarrow-1$ and $\rho_{1+} \rightarrow 0$. So far, we have been able to explore this issue only through simulations. As Fig. 5 shows, it can be tightly bound to either edge of the gap, or be isolated from both. In this case, we varied only the mean (of Gaussian $\mathbb{X}$ with variance 1/4), from 0 to 0.5 (corresponding to the cases of Fig. 3 and 1 , respectively). We see that $\lambda_{1-}$ moves from being bound to 0 , through being detached at intermediate values and to being bound near -1 . Indeed, the distribution near the right edge appears to be a pure exponential. On closer examination, the weight of this component appears to be less than unity, indicating that $\lambda_{1-}$ is found to be positive for some realizations of $\mathbb{X}$. It is unclear if this feature persists as $N \rightarrow \infty$; further studies are 


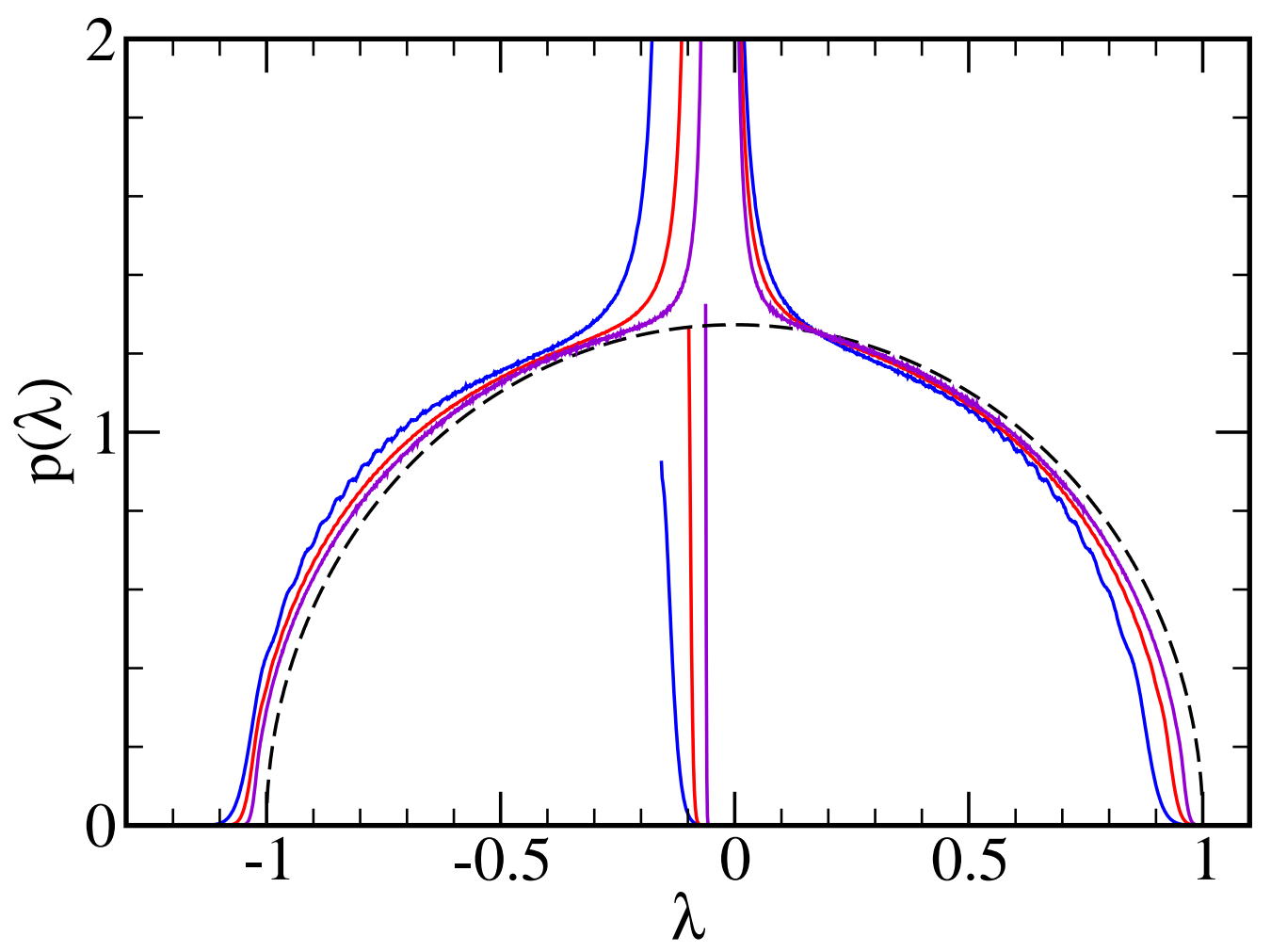

Figure 6. Scaled eigenvalue probability distribution functions of $2 N \times 2 N$ random split graph adjacency matrices with I-E connection probability 0.5 and $N=40$ (blue), $N=100$ (red) and $N=250$ (violet). Black dashed line shows limiting semi-circle distribution. Note the almost vertical line at the left edge of each of the gaps indicate that there is one eigenvalue "tightly bound" to the left edge in these cases.

underway to explore if the exponential distribution here approaches a universal limit. In the language of interactions between eigenvalues, the interpretation would be that $\lambda_{1-}$ experiences a constance attractive force from a wall (formed by the the bulk eigenvalues). However, the potential associated with such a wall is finite, as $\lambda_{1-}$ is able to penetrate into the wall with non-zero probability. As the mean increases, the distribution of the detached $\lambda_{1-}$ appears to be more Gaussian-like, with noticeable asymmetry. A possible conjecture is that, as $N \rightarrow \infty$, the width of this Gaussian decreases, resulting in a $\delta$-like distribution. In the last case with mean 0.5 , the distribution is reminiscent of a critical point associated with an unbinding transition: with $\ln p$ being linear far from the wall, but quadratic near -1. Clearly, many questions arise and will provide fertile grounds for future research. 


\section{Summary and outlook}

With application in a wide range of fields, ensembles of random matrices and the pdf of their associated spectra are fascinating topics of study. For example, in network science and graph theory, adjacency matrices of random networks/graphs form such ensembles. In this paper, we focus on the connection between ensembles of bipartite graphs and the related split graphs. Our motivation comes from social networks, where bipartite graphs describe the contacts between two otherwise totally disjoint subgroups, while split graphs correspond to connecting all members of one of the two subgroups (to form a "clique"). Simulations show several striking features in the epdf which result from adding such contacts, especially the emergence of a gap between -1 and 0 in the spectra. ${ }^{*}$ Our conclusion is that, though nearly all of these features can be understood, many lines of inquiry remain open. We end by listing a few here.

Much of our analysis can be improved, both in terms of rigor and accuracy beyond the leading order. The most pressing issue is a better understanding of the location and distribution of the gap eigenvalue. The language of interacting eigenvalues should be explored in this context, so as to clarify if there are phase transitions like unbinding and if there are anomalous properties. It is known that certain behavior is universal in the large $N$ limit. Some preliminary data, presented in Fig. 6, show the convergence of the bulk distribution, as $N$ increases, towards a semi-circle. What are the finite size effects? and is there finite size scaling? Work in this direction is in progress and the results will be interesting regardless of what they show. By contrast, we know only of the presence of the gap eigenvalue, while much of the details of the location and distribution remain to be explored. Beyond the issues associated with i.i.d. elements in our $\mathbb{X}$ 's, a much more challenging problem is the effects of correlations. In our original model of extreme introverts and extroverts, the dynamic rules are simple: choose an individual at random and let it cut/add a random link. Yet, the resulting ensemble of adjacency matrices, $\mathcal{P}\left(\mathbb{A}_{X I E}\right)$, contains highly correlated elements[35]. Indeed, as the numbers of one subgroup overtake those of the other, there is an extraordinary transition [32, 38]. At the transition (when the numbers are the same), there are giant fluctuations in $\mathbb{X}$, specifically, $\langle u|\mathbb{X}| u\rangle=\sum_{i j} X_{i j}$ being equally likely to be in the entire range $\left(0, N^{2}\right)$. How are such giant fluctuations and correlations reflected in the epdfs of the adjacency matrices? Preliminary data show qualitatively the same features as in Fig. 11 [44], but a systematic study may find novel and interesting behavior. Further, while the studies here involve explicitly known ensembles, the more realistic models of social networks involve introverts and extroverts who "prefer" generic, finite degrees. In that case, the dynamics typically violates detailed balance, so that the stationary distributions of the adjacency matrices are not known analytically[34]. What features are displayed in those epdfs? and can we understand them, whether they belong to the same universality classes or not? Such questions take us to the vast and unfamiliar territory of non-equilibrium statistical mechanics, with seemingly unlimited and novel phenomena to be discovered.

* Though our study was based on the symmetric case where the numbers in the two subgroups are the same $\left(N_{1}=N_{2}\right)$, the generalization to asymmetric cases is straightforward, with similar conclusions. The only difference is that all the random matrices will display a null space of dimension $\left|N_{1}-N_{2}\right|$. 


\section{Acknowledgements}

One of us (RKPZ) has benefited much from numerous discussions with John Cardy, over many years, on various topics in theoretical physics. It is a great pleasure for us to contribute this article to a special issue of this journal in honor of his 70th birthday. This research is support in part by the United States NSF through grant DMR-1507371. This study germinated during the authors' stay at the MPIPKS in 2013-2014, under the auspices of an ASG. The hospitality of Frank Jülicher is gratefully acknowledged. Finally, we thank Uwe Täuber for inviting us to write an article for this occasion, as well as Zoltán Toroczkai and Mohammadmehdi Ezzatabadipour for illuminating discussions.

\section{References}

[1] C. E. Porter, Statistical theories of spectra: Fluctuations, (Academic, New York, 1965).

[2] J. Wishart, Biometrika 20A, 32 (1928).

[3] R. M. May, Nature (London) 238413 (1972).

[4] R. M. May, Stability and Complexity in Model Ecosystems (Princeton University Press, Princeton, 1973).

[5] P. J. Forrester, N. C. Snaith, and J. J. M. Verbaarschot, J. Phys. A: Math. Gen. 36, R1 (2003).

[6] P. F. Forrester, Log-Gases and Random Matrices, (Princeton University Press, Princeton, 2010).

[7] E. Wigner, Ann. of Math. 62, 548 (1955).

[8] E. Wigner, Ann. of Math. 67, 325 (1958).

[9] L. Arnold, Z. Wahrscheinlichkeitstheorie und Verw. Gebiete 19, 191 (1971).

[10] V. A. Marchenko, and L. A. Pastur, Mat. Sb. N.S. (in Russian) 72, 507 (1967).

[11] F. Benaych-Georges and R. R. Nadakuditi, Adv. Math. 227, 494 (2011).

[12] S. O'Rourke and P. M. Wood, Ann. Inst. H. Poincaré Probab. Statist. 53, 1241 (2017).

[13] F. Cheung, Spectral Graph Theory, (no. 92 in CBMS Regional Conference Series. Conference Board of Mathematical Sciences, 1994).

[14] J.N. Bandyopadhyay and S. Jalan, Phys. Rev. E 76, 026109 (2007).

[15] P. Mieghem, Graph Spectra for Complex Networks, (Cambridge University Press, New York, 2011).

[16] R. R. Nadakuditi and M. E. J. Newman, Phys. Rev. Lett. 108, 188701 (2012).

[17] T. P. Peixoto, Phys. Rev. Lett. 111, 098701 (2013).

[18] B.D. MacArthur, R.J. Sánchez-García, and R.W. Anderson, Discrete Appl. Math. 156, 3525 (2008).

[19] A. Nyberg, T. Gross, and K. E. Bassler, J. Comp. Networks 3, 543 (2015).

[20] C.P. Dettmann and G. Knight, preprint arXiv:1704.00640 (2017).

[21] A. Arenas, A. Díaz-Guilera, J. Kurths, Y. Moreno, and C. Zhou, Phys. Rep. 469, 93 (2008).

[22] B.D. MacArthur, and R.J. Sánchez-García, Phys. Rev. E 80, 026117 (2009).

[23] F. Chung, L. Lu, and V. Vu, Proc. Natl. Acad. Sci. U.S.A. 100, 6313 (2003).

[24] D.W. Lang, Phys. Rev. 135, B1082 (1964).

[25] R.C. Jones, J.M. Kosterlitz, and D.J. Thouless, J. Phys. A 11, L45 (1978).

[26] K. E. Bassler, P. J. Forrester, and N. E. Frankel, J. Math. Phys. 50, 033302 (2009).

[27] P. Erdős and A. Rényi, Publicationes Mathhematice (Debrecen) 6, 290 (1959).

[28] Z. Füredi and J. Komlós, Combinatorica 1, 233 (1981).

[29] T. Rogers, I. P. Castillo, R. Kühn, and K. Taked, Phys. Rev. E 78, 031116 (2008).

[30] I. J. Farkas, I. Derényi, A.-L. Barabási, and T. Vicsek, Phys. Rev. E 64, 026704 (2001).

[31] R. K. P. Zia, W. Liu, S. Jolad, and B. Schmittmann, Phys. Proc. 15, 102 (2011).

[32] R. K. P. Zia, W. Liu, and B. Schmittmann, Phys. Proc. 34124 (2012).

[33] W. Liu, B. Schmittmann, and R. K. P. Zia, Europhys. Lett. 100, 66007 (2012).

[34] W. Liu, S. Jolad, B. Schmittmann, and R. K. P. Zia, J. Stat. Mech. P08001 (2013). 
[35] W. Liu, B. Schmittmann, and R. K. P. Zia, J. Stat. Mech. P05021 (2014).

[36] S. Földes, and P. L. Hammer, Canad. J. Math. 29, 666 (1977).

[37] R. Tyshkevich, and A. A. Chernyak, Vesti Akad. Navuk BSSR Ser. Fiz.-Mat. Navuk 5, 14 (1979).

[38] K. E. Bassler, W. Liu, B. Schmittmann, and R. K. P. Zia, Phys. Rev. E 91, 042102 (2015).

[39] K. E. Bassler, D. Dhar, and R. K. P. Zia, J. Stat. Mech. P07013 (2015).

[40] P.W. Holland, K.B. Laskey, and S. Leinhardt, Soc. Networks 5, 109 (1983).

[41] S.E. Fienberg, M.M. Meyer, and S.S. Wasserman, J. Am. Stat. Assoc. 80, 51 (1985).

[42] K. Faust and S.S. Wasserman, Soc. Networks 14, 5 (1992).

[43] K. E. Bassler, P. J. Forrester, and N. E. Frankel, J. Math. Phys. 51, 123305 (2010).

[44] M. Ezzatabadipour, K.E. Bassler, and R.K.P. Zia, unpublished.

\section{Appendix}

Here, we consider the exceptions to the generic case detailed in Section 2.

\subsection{Orthogonality}

If some of the eigenvector of $\mathbb{W}$ are orthogonal to $|u\rangle$ (denoted by $\left|w_{o}\right\rangle$ ), then $c_{o}=0$ and Eqn. (11) implies that $|v\rangle$ is just $\left|w_{o}\right\rangle$ with $\lambda_{o \pm}=\rho_{o \pm}$. Clearly, these are the cases where $c=0$. The associated eigenvectors for $\mathbb{A}_{S}$ are the doublets $\mathbb{W}\left|w_{o}\right\rangle / \lambda_{o \pm},\left|w_{o}\right\rangle$. This leaves an orthogonal subspace spanned by $c \neq 0$ vectors, which we label by $\left|w_{\gamma}\right\rangle ; \gamma=1, \ldots, L<N$. Following the same route as above, we have

$$
\mathbb{W}\left|w_{\gamma}\right\rangle=\mu_{\gamma}\left|w_{\gamma}\right\rangle ; c_{\gamma}=\left\langle u \mid w_{\gamma}\right\rangle \neq 0
$$

and we can decompose

$$
\mathbb{W}=\mathbb{W}_{o}+\mathbb{W}_{\gamma} \equiv \sum_{o} \mu_{o}\left|w_{o}\right\rangle\left\langle w_{o}\left|+\sum_{\gamma} \mu_{\gamma}\right| w_{\gamma}\right\rangle\left\langle w_{\gamma}\right|
$$

Within this subspace, we again have

$$
\mathbb{W}_{\gamma}|v\rangle=\left(\lambda^{2}+\lambda\right)|v\rangle-\lambda c|u\rangle
$$

The rest of the analysis is now clear and and (12) now reads

$$
1=\lambda \sum_{\gamma=1}^{M} \frac{c_{\gamma}^{2}}{\lambda^{2}+\lambda-\mu_{\gamma}}
$$

leading us to the rest of the $2 L$ eigenvalues and eigenvectors of $\mathbb{A}_{S}$.

In the special case that all but one $\left|w_{\alpha}\right\rangle$ is orthogonal to $|u\rangle$, then $|u\rangle$ itself must also be an eigenvector. In that case, let us denote $\mathbb{W}|u\rangle=\mu_{u}|u\rangle$ which leads us to

$$
\mathbb{W}|u\rangle=\left(\lambda_{u}^{2}+\lambda_{u}-\lambda_{u} N\right)|u\rangle
$$

and

$$
\lambda_{u \pm}=\frac{1}{2}\left\{N-1 \pm \sqrt{(N-1)^{2}+4 \mu_{u}}\right\}
$$

The reduction to $N=1$ is trivial, as $\mathbb{A}_{B} \equiv \mathbb{A}_{S}$ and $\lambda_{ \pm} \equiv \eta_{ \pm}$! Also, it means that the average of the elements of $\mathbb{W}$ is just $\mu_{u} / N$, since that average is $\sum_{i j} W_{i j} / N^{2}=\langle u|\mathbb{W}| u\rangle / N^{2}$. 


\subsection{Null space}

Suppose $K$ of the $\mu$ 's are zero (corresponding to $2 L \eta$ 's), denoted by $\mu_{\kappa}=0 ; \kappa=1, \ldots, K<N$. and associated with eigenvectors $\mathbb{W}\left|w_{\kappa}\right\rangle=0$. Since $\mathbb{W}=\mathbb{X}^{T} \mathbb{X}$, we must have $\mathbb{X}\left|w_{\kappa}\right\rangle=0=$ $\mathbb{X}^{T}\left|\tilde{w}_{\kappa}\right\rangle$, where $\left|\tilde{w}_{\kappa}\right\rangle$ is the transpose of the left eigenvector of $\mathbb{X}$. In other words, the doublets for $\mathbb{A}_{B}$ associated with $\eta_{\kappa \pm}=0$ are $\left(|0\rangle,\left|w_{\kappa}\right\rangle\right)$ and $\left(\left|\tilde{w}_{\kappa}\right\rangle,|0\rangle\right)$. Now, these $\eta$ 's lead us to $\rho_{\kappa^{+}}=0$ and $\rho_{\kappa^{-}}=-1$. The first naively imply the pole terms there are absent (as its residue is $\left.\rho_{\kappa+}\right)$. However, its treatment is similar to the orthogonal cases. In particular, we simply verify that the doublets $\left(\left|\tilde{w}_{\kappa}\right\rangle,|0\rangle\right)$ is in the null space of $\mathbb{A}_{S}$, i.e., they are the eigenvectors of $\mathbb{A}_{S}$ associated with $\lambda_{\kappa+}=0$. As for $\rho_{\kappa_{-}}=-1$, the pole terms are non-singular and the treatment for them remains the same as above. This subsection also addresses the issue when $\lambda$ vanishes.

\subsection{Degeneracy}

If there is a set of eigenvectors, $\left|w_{s}\right\rangle$, with the same eigenvalue $\mu_{s}$, then the only point is that the residue of the poles at $\rho_{s \pm}$ becomes $\Sigma_{s} c_{s}^{2}=\left\langle u\left|\left[\Sigma_{s}\left|w_{s}\right\rangle\left\langle w_{s}\right|\right]\right| u\right\rangle=\left\langle u\left|\square_{s}\right| u\right\rangle$, where $\mathbb{\square}_{s}$ is the unit matrix within the subspace spanned by $\left\{\left|w_{s}\right\rangle\right\}$. Thus, it is invariant to rotations within that subspace, i.e., $\Sigma_{s} c_{s}^{2}$ does not depend on the precise choice of the set $\left\{\left|w_{s}\right\rangle\right\}$. 